\title{
Artefact
}

Techniques, histoire et sciences humaines

\section{Anne-Laure Carré et Sophie Lagabrielle (dir.), Flacons, fioles et fiasques : de l'Antiquité à nos jours}

Actes du troisième colloque international de l'association Verre \& Histoire, Rouen-Vallée de la Bresle, 4-6 avril 2013, Paris, Verre \& Histoire, 2019

\section{Lætitia Zicavo}

\section{(2) OpenEdition}

\section{Journals}

\section{Édition électronique}

URL : http://journals.openedition.org/artefact/6096

DOI : 10.4000/artefact.6096

ISSN : 2606-9245

Éditeur :

Association Artefact. Techniques histoire et sciences humaines, Presses universitaires du Midi

\section{Édition imprimée}

Date de publication : 15 juillet 2020

Pagination : 351-354

ISBN : 978-2-8107-0691-4

ISSN : 2273-0753

Référence électronique

Lætitia Zicavo, «Anne-Laure Carré et Sophie Lagabrielle (dir.), Flacons, fioles et fiasques : de l'Antiquité à nos jours », Artefact [En ligne], 12 | 2020, mis en ligne le 21 décembre 2020, consulté le 23 décembre 2020. URL : http://journals.openedition.org/artefact/6096; DOI : https://doi.org/10.4000/artefact. 6096

Ce document a été généré automatiquement le 23 décembre 2020.

\section{c) (†)}

Artefact, Techniques, histoire et sciences humaines est mise à disposition selon les termes de la Licence Creative Commons Attribution - Pas d'Utilisation Commerciale - Pas de Modification 4.0 International. 


\section{Anne-Laure Carré et Sophie Lagabrielle (dir.), Flacons, fioles et fiasques : de l'Antiquité à nos jours}

Actes du troisième colloque international de l'association Verre \& Histoire, Rouen-Vallée de la Bresle, 4-6 avril 2013, Paris, Verre

\& Histoire, 2019

Lætitia Zicavo

\section{RÉFÉRENCE}

Anne-Laure Carré et Sophie Lagabrielle (dir.), Flacons, fioles et fiasques : de l'Antiquité à nos jours, actes du troisième colloque international de l'association Verre \& Histoire, Rouen-Vallée de la Bresle, 4-6 avril 2013, Paris, Verre \& Histoire, 2019, 232 pages.

1 L'ouvrage Flacons, fioles et fiasques présente les actes du troisième colloque international de l'association Verre \& Histoire qui s'est tenu du 4 au 6 avril 2013 à Rouen et dans la Vallée de la Bresle. Ce large colloque a réuni pas moins de cent dix personnes dont vingt-cinq communicants et auteurs et suscité quatre expositions à Rouen et Martainville. Cet ouvrage collectif richement illustré rassemble dix-sept contributions.

2 L'introduction d'Anne-Laure Carré et Sophie Lagabrielle explique l'objectif de cette étude: démontrer la très grande adaptabilité culturelle et fonctionnelle de l'objet "flacon »; souligner sa capacité à profiter des innovations techniques et industrielles et illustrer son réel "pouvoir de séduction" dans une économie de marché. Elles rappellent qu'un flacon est essentiellement reconnaissable par sa petite taille même s'il a une indéniable pluralité de formes et de fonctions. L'ouvrage prend en compte différentes morphologies du flacon et différents dérivés : le carafon, l'urinal, le pot, l'alambic entre autres. L'ouvrage propose une approche chronologique sur le temps long du flacon dans sa dimension matérielle mais aussi symbolique. Il se découpe en 
trois parties: "période antique », « Moyen Âge et Renaissance » et "époque contemporaine ».

3 Les différentes contributions de cet ouvrage renouvellent l'approche de la thématique du contenant en utilisant diverses sources. Si les apports de l'archéologie sont toujours précieux, l'histoire matérielle peut se tourner vers les inventaires de boutiques dès le $\mathrm{XVIII}^{\mathrm{e}}$ siècle ainsi que les traités techniques et les monographies d'entreprises. L'iconographie est riche en matériaux pour le chercheur qui se penche sur les enluminures des manuscrits médiévaux. Enquêtes de terrain et presse féminine complètent les sources d'une histoire vivante.

apport essentiel de ce colloque est de démontrer la pertinence du rapport contenant-contenu. La première partie de l'ouvrage, consacrée à la période antique, s'ouvre ainsi sur une première étude illustrant la volonté d'écrire l'histoire conjointe des parfums et flacons à l'époque romaine impériale en examinant le rapport entre les huiles parfumées, les cosmétiques et les flacons (Jean-Pierre Brun). Dès l'époque grécoromaine, sont fabriqués des flacons à la fois utilitaires et astucieux pour le transport et le stockage. Une évolution progressive des matériaux est à observer, même si la place $\mathrm{du}$ verre dans la fabrication des flacons à fards notamment reste prépondérante (Philippe Walter). Si l'origine du verre reste difficile à établir avec précision (Caroline Dorion-Peyronnet), l'adaptation de ce matériau à l'innovation technique est une caractéristique indéniable. S'agissant à l'origine d'un objet artisanal, le flacon devient un support manifeste à cette innovation. Les premiers flacons sont obtenus par une technique développée au Proche-Orient il y a plusieurs milliers d'années. L'invention du soufflage $\mathrm{au} \mathrm{I}^{\mathrm{er}}$ siècle avant notre ère a permis la réduction des coûts de fabrication. D'autre part, ce récipient, au fil des siècles, revêt de nouvelles symboliques ou sert de nouveaux usages. Ainsi, durant l'Antiquité, le flacon est associé à la sphère du sacré et fait partie des rituels d'inhumation. L'étude de la nécropole des Cordiers à Mâcon souligne des pratiques funéraires diverses. Des balsamaires ont été retrouvés brisés volontairement dans des fosses-dépotoirs : leur contenu pouvait être utilisé lors du bûcher funèbre (Aline Colombier-Gougouzian). Une étude chronologique de la verrerie antique de Haute-Normandie du $\mathrm{I}^{\mathrm{er}}$ au $\mathrm{v}^{\mathrm{e}}$ siècle démontre une évolution à la fois fonctionnelle et morphologique des objets en verre (Yves-Marie Adrian). Enfin, il est important de considérer la question du vocabulaire latin, assez pauvre malgré les très nombreuses catégories de récipients. Si le terme d'unguentarium revêt un sens général et est utilisé par les archéologues pour désigner un large éventail de flacons, le balsamaire est un synonyme moderne du premier terme, création récente relevant du jargon archéologique et antiquaire (Paul Fontaine et Chantal Fontaine-Hodiamont). Cet ouvrage met par conséquent en avant l'importance des découvertes archéologiques qui permettent une meilleure connaissance de la géographie ancienne du verre, de l'évolution des matériaux et des usages.

5 La période du Moyen Âge et de la Renaissance est traitée dans une deuxième partie plus réduite (quatre contributions contre six ou sept dans les deux autres parties). Au cours de cette période, l'usage des récipients de petite taille se diversifie, comme le montrent plusieurs sources écrites, iconographiques et matérielles. À l'inverse de l'âge romain, les contenants du Moyen Âge ont des dimensions réduites et aucun systématisme n'est à observer dans leurs formes (Sophie Lagabrielle). À partir de l'essor économique des $\mathrm{XII}^{\mathrm{e}}$ et $\mathrm{XIII}^{\mathrm{e}}$ siècles, les "sachants ", tels les apothicaires et médecins, s'emparent de l'urinal qui devient un outil indispensable pour le diagnostic. Cet objet pansu et doté 
d'un col est particulièrement important dans le cadre médical en Occident médiéval, la preuve en est que le récipient occupe une place centrale dans les représentations de la scène de consultation. Très codifié, cet examen devient progressivement un jeu de rôles lors duquel le patient peut être représenté par un tiers (Laurence Moulinier-Brogi). L'utilisation du verre est notamment préconisée par les alchimistes comme matière idéale des récipients (Antoine Calvet). Ses qualités sont reconnues par ailleurs à travers la verrerie pharmaceutique dont les quantités augmentent dès le début $d u \mathrm{XVI}^{\mathrm{e}}$ siècle. Sa transparence, son imperméabilité et sa composition chimiquement inerte font du verre un matériau parfait dans l'apothicairerie (Danièle Alexandre).

6 Les contributions de la troisième partie de l'ouvrage, consacrée à la période contemporaine, participent d'une meilleure connaissance des lieux de production de verrerie, des stratégies de vente des parfums et de leurs contenants ainsi que de la recherche esthétique autour de cet objet. Ce troisième volet inclut un panorama des sites de production verrière utilitaire aux États-Unis du milieu du XVII ${ }^{e}$ au XIX ${ }^{e}$ siècle. Si le concept de flasques décoratives vient d'une tradition européenne, celles-ci s'adaptent au goût américain notamment avec les figured flasks qui représentent une part importante des bouteilles de liqueur décoratives de petite taille (Alice Cooney Frelinghuysen). Le nord de la France regorge aussi de régions de tradition verrière ancienne comme la grande Thiérache (Stéphane Palaude) et l'Orne (Matthieu Le Goïc et Odile Leconte). Le flacon, toujours associé étroitement au parfum, se pose également en instrument de stratégie commerciale. Déjà au XvIII siècle, l'activité de la parfumerie, qui connaît un essor fulgurant grâce à la cour de Versailles, développe des produits en lien avec le parfum tels que les flaconniers ou caves à parfum et divers instruments liés à leur utilisation, parfois rassemblés dans des nécessaires (Annick Le Guérer). Objet de marketing, le flacon lui-même est un des enjeux d'une nouvelle bataille mercantile qui s'instaure au XIX siècle : l'évolution des prix, l'importance croissante du visuel et le jeu rhétorique dans la stratégie commerciale du parfumeur caractérisent désormais le marché de la parfumerie (Eugénie Briot). L'intérêt croissant du marchand pour ses flacons se traduit par l'organisation de devantures de plus en plus aérées qui mettent en valeur l'objet « flacon ». Les parfumeurs de luxe délaissent le style art nouveau pour un choix volontaire de sobriété. Néanmoins, quelques créations plus audacieuses apparaissent au début $\mathrm{du} \mathrm{xx}^{\mathrm{e}}$ siècle. Les grands parfumeurs sont alors tentés par la figuration artistique et font appel à des créateurs comme René Lalique par exemple (Rosine Lheureux). Celui-ci est une figure importante du milieu du flaconnage et un industriel de talent. Il associe à la fabrication en série et aux techniques de production mécanique une recherche esthétique. Il applique un souci particulier au travail du bouchon qui devient une véritable œuvre : ses bouchons-sculptures renvoient le flacon au rôle de piédestal. La mise en scène et l'esthétique du flacon sont ainsi des arguments commerciaux qui participent d'une nouvelle conception de la parfumerie au $\mathrm{xx}^{\mathrm{e}}$ siècle (Véronique Brumm).

7 Finalement, le flacon est un contenant utile mais aussi décoratif. C'est un marqueur des niveaux socio-économiques. Si sa fabrication a pu être démocratisée par l'évolution des techniques, il s'insère dans l'économie domestique et professionnelle dès l'Antiquité puis au Moyen Âge. Il se lie au développement moderne de l'hygiène et aux soins de toilette au XVIII ${ }^{\mathrm{e}}$ siècle. Au XIX ${ }^{\mathrm{e}}$ siècle, devenu objet ordinaire, il s'adapte aux inflexions du marché, puis s'inscrit avec succès dans les industries du luxe, notamment français, $\mathrm{au} \mathrm{xx}^{\mathrm{e}}$ siècle. Un tel sujet d'étude suggère une pluralité d'axes de recherche. Faire 
l'histoire des flacons, fioles et fiasques, c'est faire à la fois une histoire de matériaux, une histoire d'objets et une histoire d'emballages (Denis Woronoff). Ces actes de colloque proposent une approche sur le temps long de l'histoire d'un contenant millénaire, un contenant aux noms, formes et usages pluriels.

\section{AUTEURS}

\section{LAETITIA ZICAVO}

Laboratoire Identités, Cultures, Territoires (ICT EA 337/Université de Paris) 